\title{
Cytological Analysis of the Parasexual Cycle in Dictyostelium discoideum
}

\author{
By T. BRODY* AND K. L. WILLIAMS \\ Genetics Laboratory and Department of Biochemistry, University of Oxford, \\ Oxford $O X_{\mathrm{I}} 3 Q U$
}

(Received 19 November 1973; revised 8 January 1974)

\section{SUMMARY}

We have developed a rapid and simple technique for examining chromosomes of the cellular slime mould Dictyostelium discoideum, based on standard cytological techniques involving fixation in methanol/acetic acid and staining with Giemsa stain. The haploid chromosome set is analysed. Four diploid strains are examined cytologically in order to determine their ploidal stability and to determine the pathway of haploidization in the parasexual cycle. In the absence of cloning, diploid strains eventually revert to haploid. Haploidization proceeds via transient aneuploidy. Aneuploids of all classes, $n+I$ through to $2 n+2$, have been demonstrated cytologically. It is concluded that aneuploids can divide but that they are unstable and readily lose chromosomes to form aneuploids of lower chromosome numbers and eventually haploids. Hence, the process of haploidization is similar to that found in the parasexual cycle of Aspergillus nidulans.

\section{INTRODUCTION}

The cellular slime mould Dictyostelium discoideum is normally a haploid organism, although diploid strains have been isolated from haploid laboratory cultures (Ross, 1960). Although parasexual changes in ploidy have recently been used for the genetic analysis of $D$. discoideum (Katz \& Sussman, 1972; Williams, Kessin \& Newell, 1974), there has been no satisfactory cytological confirmation of parasexuality. There have been several reports describing the chromosomes (Ross, 1960; Sussman, I961), but a major problem has been the absence of an easy, rapid method for obtaining good fixed and stained cytological preparations.

We report here a quick reliable fixation and staining procedure for producing cytological preparations of Dictyostelium discoideum. This procedure relies upon the standard procedures for preparation of mammalian chromosomes (Hamerton, I97I). We have studied the stability of haploid and diploid strains and the haploidization of diploid strains via aneuploidy. These aspects of the parasexual cycle have been analysed previously, but as yet there is no consensus concerning ploidal stability or the mechanism of haploidization (Ross, 1960; Sinha \& Ashworth, I969; Katz \& Sussman, 1972).

\section{METHODS}

Growth of Dictyostelium discoideum. Stocks of spores of all strains were stored in silica gel at $4{ }^{\circ} \mathrm{C}$ (Perkins, 1962). Details of the strains studied and their origin are given in Table $\mathrm{I}$. All amoebae used were derived from clonal plates growing in association with Aerobacter aerogenes. Amoebae were scraped from the edge of a clone containing approximately $10^{7}$

* Present address: Genetics Department, The Hebrew University of Jerusalem, Jerusalem, Israel. 
Table I. Description of the strains of $D$. discoideum used in this study

Strains denoted NP are haploid, DP are diploid, and $\mathrm{x}$ are reassorted haploids containing chromosomes from both parents of a diploid. All of these strains were derived from strain NC4 and hence are mating type $A_{1}$ (Erdos et al. 1973). All form normal spores, or, when mixed with strain VI2 (mating type $A_{2}$ ), readily form macrocysts. No attempts have been made to induce diploids to form macrocysts.

\begin{tabular}{|c|c|c|c|c|c|}
\hline \multirow[b]{2}{*}{ Strain } & \multirow[b]{2}{*}{$\begin{array}{l}\text { Parent } \\
\text { strain }\end{array}$} & \multirow{2}{*}{ Source } & \multicolumn{3}{|c|}{$\begin{array}{l}\text { Phenotype (where appropriate the } \\
\text { genotype is shown in brackets) }\end{array}$} \\
\hline & & & Pigment* & $\begin{array}{c}\text { Growth at } \\
\text { restrictive } \\
\text { temperature } \\
\left(27^{\circ} \mathrm{C}\right) \dagger\end{array}$ & $\begin{array}{c}\text { Axenic/ } \\
\text { non-axenic } \\
\text { growth }\end{array}$ \\
\hline AX3 & $\mathrm{NC4}$ & Loomis, 1971 & + & + & Axenic $(\mathrm{Ax} 3)$ \\
\hline NPI 6 & $\mathrm{AX} 3$ & $\ddagger$ & + & $-($ tsgB3) & Axenic $(\mathrm{A} \times 3)$ \\
\hline NP2 & $\mathbf{A} \times 3$ & & + & - (tsgAI) & Axenic (AX3) \\
\hline M28 & - & $\begin{array}{l}\text { Katz \& } \\
\text { Sussman, } 1972\end{array}$ & bwn & - (tsgeI 3) & Non-axenic \\
\hline X2 & DP3 & $\ddagger$ & bwn & $-($ tsgAI $)$ & Axenic (AX3) \\
\hline DP3 & M28, NP2 & $\ddagger$ & $+(+/$ bwn $)$ & $\begin{array}{c}+(\operatorname{tsgeI} 3 /+ \\
\left.+/ t_{\text {sgAI }}\right)\end{array}$ & $\begin{array}{l}\text { Non-axenic } \\
(-/ \mathbf{A} \times 3) \S\end{array}$ \\
\hline NPI 7 & $\mathbf{x} 2$ & $\ddagger$ & bwn & $-(\operatorname{tsg} A I)$ & Axenic $\left(\mathrm{Ax}_{3}\right)$ \\
\hline $\begin{array}{l}\text { NP24 } \\
\text { (formerly } \\
\text { Ax-2g88) }\end{array}$ & $\mathbf{A X 2}$ & $\begin{array}{l}\text { Gingold and } \\
\text { Ashworth, } \\
\text { Leicester }\end{array}$ & + & $-($ tsg-I 4$)$ & Axenic (AX2) \\
\hline DP26 & $\mathrm{NP24}, \mathrm{X} 2$ & $\ddagger$ & $+(+/$ bwn $)$ & $\begin{array}{c}+(\text { tsg-I } 4 /+ \\
+/ \text { tsgAI })\end{array}$ & $\begin{array}{l}\text { Axenic } \\
(\operatorname{AX2} / \mathrm{AX} 3)\end{array}$ \\
\hline DP27 & NPI 6, X2 & $\ddagger$ & $+(+/$ bwn $)$ & $\begin{array}{l}+(\text { tsgB3 } /+ \\
+/ \text { tsgaI }^{-}\end{array}$ & $\begin{array}{l}\text { Axenic } \\
(\mathrm{A} \times 3 / \mathrm{Ax} 3)\end{array}$ \\
\hline DP28 & NP24, NPI 7 & $\ddagger$ & $+(+/$ bwn $)$ & $\begin{array}{c}+(\text { tsg-I } 4 /+ \\
+/ \text { tsgAI })\end{array}$ & $\begin{array}{l}\text { Axenic } \\
(\mathrm{A} \times 2 / \mathrm{A} \times 3)\end{array}$ \\
\hline DP29 & NP24, NPI 6 & $\ddagger$ & $+(+1+)$ & $\begin{array}{l}+(\text { tsg-I } 4 /+ \\
+/ \text { tsgB3 })\end{array}$ & $\begin{array}{l}\text { Axenic } \\
(\mathrm{A} \times 2 / \mathrm{A} \times 3)\end{array}$ \\
\hline & $\begin{array}{l}\text { Brown pigm } \\
\text { enotes wild-t } \\
\text { enotes wild- } \\
\text { tment of } B i \\
\text { c growth is }\end{array}$ & $\begin{array}{l}\text { rmation; tsg }= \\
\text { on-brown pigme } \\
\text { rowth at } 27^{\circ} \mathrm{C} \text {. } \\
\text { nistry. University } \\
\text { ssive character ( }\end{array}$ & $\begin{array}{l}\text { tation. } \\
\text { of Oxford. } \\
\text { e Williams } e\end{array}$ & ive for growth & $27^{\circ} \mathrm{C}$ \\
\hline
\end{tabular}

cells (i.e. between 20 and 25 generations of growth). Amoebae to be grown in liquid culture ( 150 cycles $/ \mathrm{min}$ at $22^{\circ} \mathrm{C}$ ) in association with $A$. aerogenes were inoculated at between $10^{4}$ and $10^{5} / \mathrm{ml}$ in a $250 \mathrm{ml}$ flask which contained $50 \mathrm{ml} \mathrm{O.OI} \mathrm{M-potassium} \mathrm{phosphate} \mathrm{pH} 6.5$ and the scrapings of $A$.aerogenes from a Petri dish of nutrient agar which had been incubated for 2 days at $22{ }^{\circ} \mathrm{C}$. Amoebae for growth in axenic medium (Watts \& Ashworth, 1970) were inoculated at $10^{4}$ to $10^{5} / \mathrm{ml}$, usually in 50 to $70 \mathrm{ml}$ axenic medium in a $250 \mathrm{ml}$ flask, and shaken at $\mathrm{I} 50 \mathrm{cycles} / \mathrm{min}$ at $22{ }^{\circ} \mathrm{C}$. The axenic medium contained $250 \mu \mathrm{g}$ dihydrostreptomycin sulphate $/ \mathrm{ml}$ to prevent the growth of $A$. aerogenes. No attempt was made to remove the bacteria upon inoculation of bacteria-fed cells into axenic medium since $A$. aerogenes was maintained clonally and streptomycin sensitivity was confirmed.

Formation of diploids. Temperature-resistant diploids were selected at a frequency of approximately $I$ in $10^{5}$ from two non-identical temperature-sensitive haploid strains (Katz \& Sussman, 1972; Williams et al. 1974). Amoebae, $5 \times 10^{6}$ of each strain, were mixed in a Linbro tissue culture dish (FB-I6-24-TC) containing I ml water and shaken at 150 cycles/ min at $22{ }^{\circ} \mathrm{C}$ for 15 to $17 \mathrm{~h}$ in the dark. The amoebae were disaggregated and plated at $\mathrm{I} \times 10^{5}$ to $3 \times 10^{5} /$ petri dish with Aerobacter aerogenes and put at the restrictive temperature 
$\left(27^{\circ} \mathrm{C}\right)$. After 3 to 4 days, temperature-resistant diploid clones appeared. Diploidy was subsequently confirmed by using spore size and genetic markers. The diploid spores were stored in silica gel at $4{ }^{\circ} \mathrm{C}$ after one or two clonal passages.

Determination of spore size. Clones were scored as haploid or diploid by comparing them with parental haploid and diploid spores by using a calibrated grid in a microscope.

Determination of plating efficiency. Amoebae were counted in a haemacytometer and diluted so that 20 to 70 cells were deposited on each agar plate which was also spread with Aerobacter aerogenes. Clones were counted after approximately 3 and 5 days at $22^{\circ} \mathrm{C}$.

Fixation procedure. Rapidly dividing amoebae, generally at a low density $\left(10^{5}\right.$ to $2 \times 10^{6} /$ $\mathrm{ml}$ ), were used for cytological examination. Between $10^{6}$ and $4 \times 10^{7}$ cells were used. Amoebae grown axenically or in association with Aerobacter aerogenes were sedimented at $150 \mathrm{~g}$ for $2 \mathrm{~min}$ and washed once with water (room temperature). The pellet of amoebae was gently resuspended in the small volume of water remaining after pouring off the supernatant fluid, then $10 \mathrm{ml}$ of freshly prepared methanol/glacial acetic acid $(3: \mathrm{I}, \mathrm{v} / \mathrm{v})$ was added dropwise initially, with shaking. The suspension was centrifuged at $150 \mathrm{~g}$ for $2 \mathrm{~min}$ and the solution poured off gently, leaving a fluffy pellet. The pellet was resuspended in methanol/acetic acid and the centrifugation step was repeated. The pellet was finally resuspended in a sufficient volume of methanol/acetic acid to give a cloudy suspension. Between two and three drops of the fixed amoebae were added to a slide previously cleaned in methanol and dried with a clean towel. The amoebae were air dried.

Staining procedure. The cells were stained with $10 \%$ Gurr's Giemsa stain (Improved R66) in $\mathrm{m} / \mathrm{I} 5$ Sorensen's phosphate buffer (pH 6.8) for Io min. The slides were examined immediately or dried and made permanent with Euparal mountant. Some slides were treated with trypsin (0.2 mg Difco trypsin/ml M/15 Sorensen's phosphate, $\mathrm{pH} 6.8$ ) for between 4 and $6 \mathrm{~min}$ before Giemsa staining. With this treatment the cytoplasmic staining was lightened and this made the inspection of the chromosomes easier.

Photography. Photomicrographs were made using bright-field optics with Kodak Microfile film in a Leica camera body fitted to a Leitz microscope.

\section{RESULTS}

Growth of Dictyostelium discoideum in axenic medium was insensitive to colchicine (levels up to $4 \mathrm{mg} / \mathrm{ml}$ were tested) and hence it was not possible to arrest amoebae at metaphase. All results were obtained by means of asynchronous cultures. The doubling time of haploid amoebae in axenic medium was Io to $\mathrm{I} 2 \mathrm{~h}$, and in liquid bacterial culture 3 to $4 \mathrm{~h}$. It was much easier to find mitotic figures in preparations of axenically grown amoebae, because the chromosomes stood out from the background and there was no ambiguity caused by the presence of bacteria, which can look similar to chromosomes. There was also a general impression that less mitotic figures were present in bacterially grown amoebae at any given time than in axenically grown amoebae, although this has not been quantitated.

\section{Characterization of cultures of haploid cells}

Two axenic strains were studied to understand the course of mitosis and characterize the chromosomal complement of normal haploid Dictyostelium discoideum. The two strains, $\mathrm{AX} 3$ and $\times 2$, are cytologically similar and the description below applies to both of them.

Early prophase was indicated by the condensation of the chromatin into long slender chromosomes, followed later by further contraction of the chromosomes that reached a 


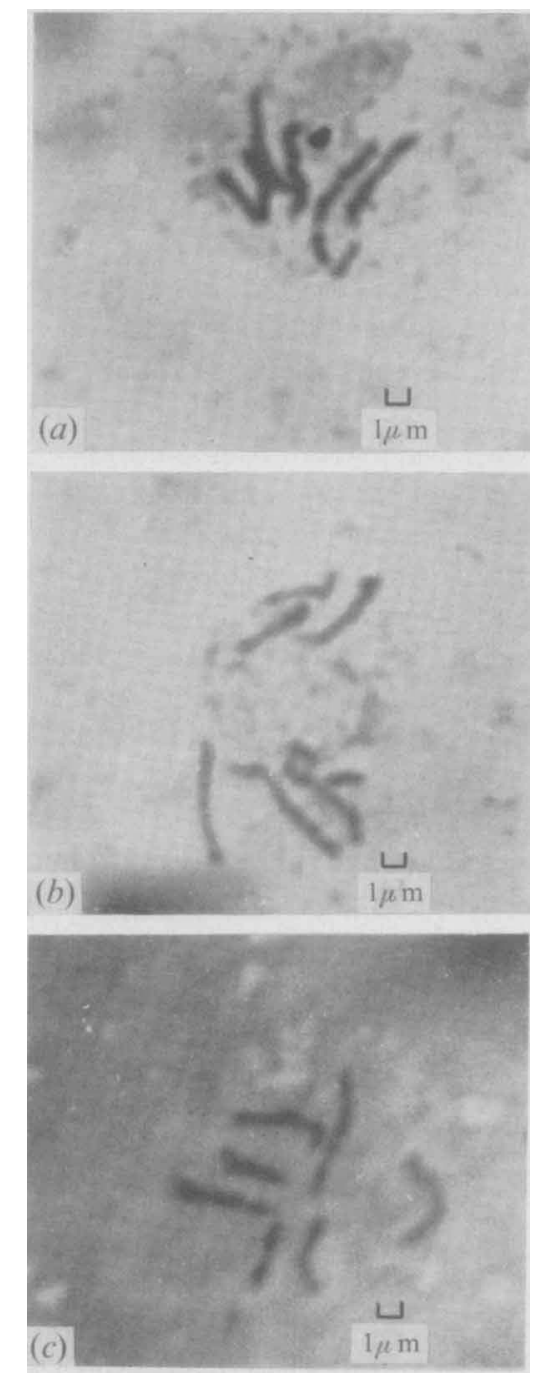

Fig. 1. Normal karyotype of $D$. discoideum. $(a),(b)$ Division figures of strains $\mathrm{X} 2$ and Ax3 respectively which contain long and short chromosomes in association with each other. There is a lightstaining region on the long chromosome which is always closest to the end associated with the short chromosome. (c) A division figure from strain $\times 2$ which contains the normal haploid number of chromosomes but which lacks the short chromosome. The long chromosome has a light-staining region. Other chromosomes also have light-staining regions in this particular cell.

maximum at metaphase. They moved into the metaphase plate and were distributed in a ring in a radial configuration suggesting that the chromosomes were acrocentric. The size of chromosomes in prophase was not much different from that of the smaller mammalian chromosomes, although they were more slender.

We observed multinucleate cells with figures in the same stage of mitosis in cultures of Dictyostelium discoideum. Often two, three, four or more prophase, metaphase, or telophase stages were seen near each other, apparently in the same cytoplasm. In observing 228 amoebae of a culture of strain $\mathrm{Ax}_{3}$ grown axenically, the ratio of uninucleate to 

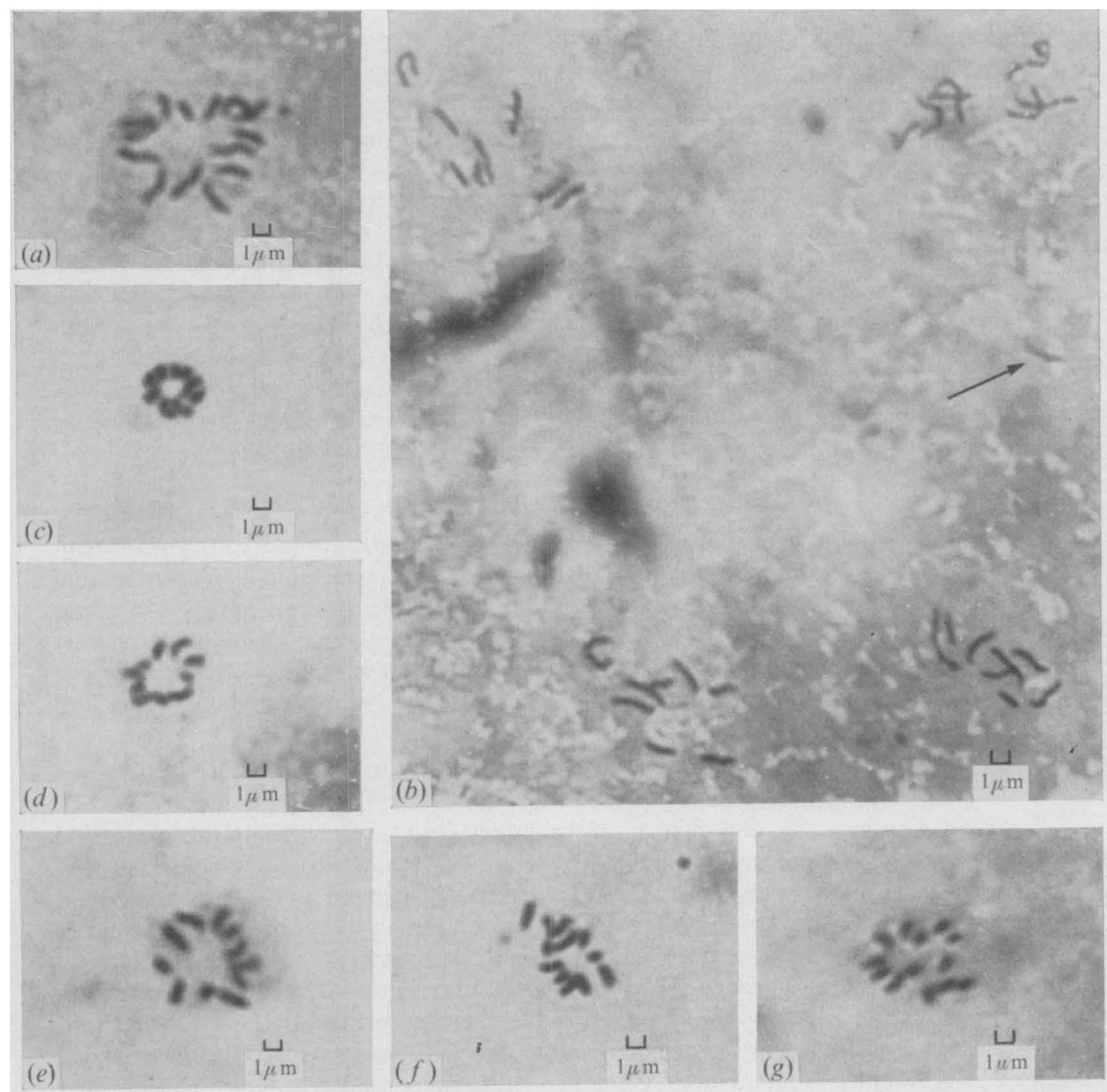

Fig. 2. Division figures present in cultures of diploid cells in the process of haploidization. (a) A diploid cell. (b) A tetranucleate cell in which each division figure contains eight chromosomes. There is an additional chromosome indicated by the arrow that is not associated with any of the other chromosomes. (c) A cell with nine chromosomes. (d) A cell with ten chromosomes. (e) A cell with I I chromosomes. The long and short chromosomes can be seen associated with each other. $(f)$ A cell with 12 chromosomes. $(g)$ A cell with 13 chromosomes.

binucleate amoebae was approximately $2: \mathrm{I}$. In other experiments the frequency of binucleate amoebae was in general less than this.

The haploid chromosome number was seven and it was found in more than $90 \%$ of all amoebae. Rarely, aneuploid cells were observed in haploid cultures. Since only a few have been observed, it was not possible to measure the relative frequency of each class, although aneuploid cells containing eight chromosomes appeared to be the most frequent. Very rare 
binucleate cells containing $6+8$ or $5+9$ chromosomes were observed. Diploid cells have been observed in cultures of both $\mathrm{AX} 3$ and $\mathrm{X} 2$ at a frequency no greater than $\mathrm{I} \%$.

Some discrimination of the chromosomes on the basis of size is possible. Figure I shows three cells in which the chromosomes were well spread. The most easily identified chromosome was long and contained a light-staining region (Figs. I $(a)$ and $(b)$ ). Often associated with the long chromosome was a short chromosome which was readily identifiable in cells in prophase. We measured the length of the long and short chromosomes in twelve wellspread cells in prophase. The ratio of long to short chromosomes was $I: 0 \cdot 28$. This difference in size decreased as the chromosomes condensed. The short chromosome was always associated with the long chromosome at the end closest to its light-staining region. The other five chromosomes were of intermediate length and were not readily distinguishable from each other. Frequently in strain $\mathrm{X2}$ the short chromosome was not present (Fig. I (c)). About half of the X2 amoebae did not have the short chromosome, although the chromosome count in these cells was still seven. More rarely the short chromosome was absent from strain Ax3. Without being able to resolve all seven chromosomes, the identity of the chromosome that replaced the short chromosome could not be determined.

\section{Characterization of cultures of diploid cells}

Four diploid strains were studied (Table I). These were DP27, produced from two temperature-sensitive haploid AX3 strains, and DP26, DP28 and DP29, produced from haploid temperature-sensitive strains of $\mathrm{AX} 2$ and AX3. All of these diploid strains can grow in axenic medium (Williams et al. 1974). When cells were taken from a single clone grown on agar in association with Aerobacter aerogenes, they were diploid on the basis of spore size and genetic markers. We have confirmed cytologically that they were diploid. A diploid cell containing 14 chromosomes is shown in Fig. 2(a).

\section{Time-course of haploidization of diploid strains}

Cytological examination. Although all of the diploid strains mentioned in this study were easily maintained by cloning, like all diploid strains of $D$. discoideum described so far, they spontaneously gave rise to amoebae that were identified as haploid on the basis of chromosome number, spore size and genetic markers. To study the process of haploidization we grew the four diploid strains both axenically and in association with bacteria, and at intervals examined the amoebae for chromosome number, spore size and plating efficiency. Figure 3 shows the results of chromosome counts after approximately 3, 7 and I 8 generations in axenic medium and for ameobae grown for 22 generations on bacteria in liquid culture after scraping from a clone. Axenically grown amoebae were studied chiefly, since it was easier to count chromosome numbers in the absence of bacteria. In all cases the initial axenic cultures were predominantly diploid but after 18 generations they were mainly haploid.

Each of the four strains were examined twice. Experiments $I$ and 2 gave similar results suggesting that, when experimental conditions are identical, relatively reproducible results can be obtained for the stability of diploid strains. The four diploid strains studied showed a similar pattern of haploidization, although it is possible that there were slight differences in stability, e.g. in both experiments DP26 was significantly less diploid after seven generations than strain DP28. However, it cannot be ruled out that this results from variability in the rates of haploidization (Sussman \& Sussman, 1963).

In Expt $\mathrm{I}$ it was suspected that diploids grown with bacteria were more stable. Hence in Expt 2 a comparison was made between diploid amoebae from the same clones cultured 


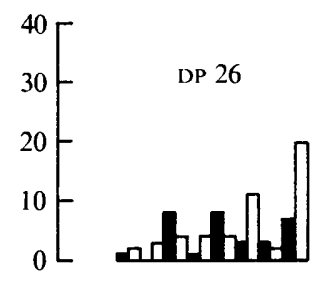

(a) Axenic, 2 to 4 generations

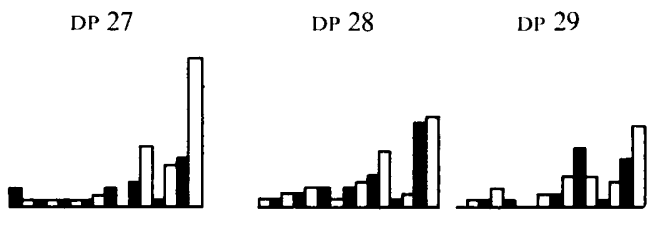

(b) Axenic, 7 to 10 generations

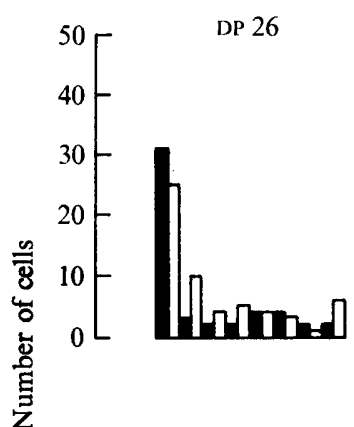

DP 26
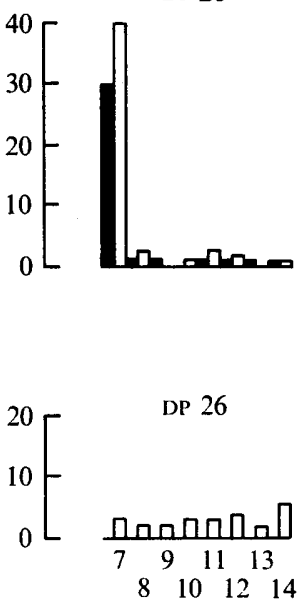

DP 27

DP 28

DP 29

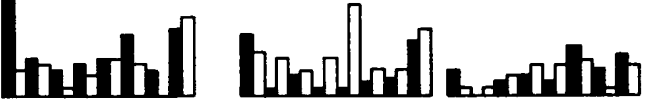

(c) Axenic, 16 to 20 generations

DP 27

DP 28

DP 29

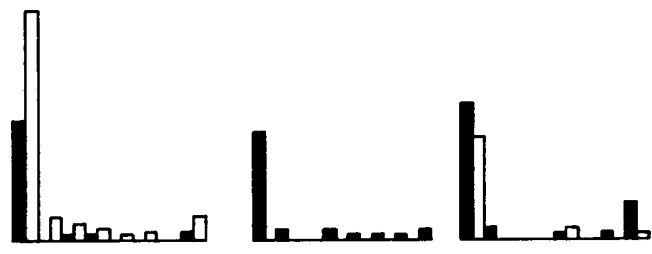

(d) Bacterial, 22 generations

DP 27

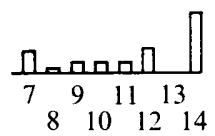

DP 28

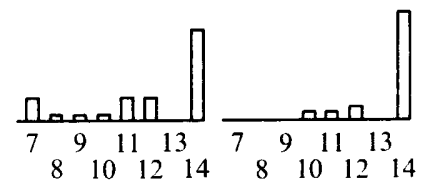

Number of chromsomes

Fig. 3. Distributions of chromosome counts per nucleus in four diploid strains of $D$. discoideum. Histograms $(a),(b)$ and $(c)$ refer to growth in liquid axenic medium. Histogram $(d)$ refers to growth on bacteria in liquid culture. Generations of growth refers to the number of generations of growth after scraping cells from a diploid clone grown on an agar plate in association with Aerobacter aerogenes. Two experiments were conducted. In Expt I (G) only results for growth in axenic medium are shown. In Expt 2 ( $\square$ ) cells from the same diploid clones were used to initiate both the axenic and bacterial cultures. Samples from the bacterial cultures were also examined at eight and 16 generations (data not shown); these were predominantly diploid. Between two and four slides were scanned systematically for each point, and attempts were made to count the chromosomes of all cells in mitosis. However, in a number of cases chromosomes overlapped making accurate counts impossible Only those counts in which there was no doubt about the chromosome number, or where the possible error involved was \pm one chromosome, are included in this figure. 
first in axenic medium and secondly in association with bacteria. A comparison of histograms of strains DP26 through to DP29 clearly suggested that diploid amoebae grown on bacteria were more stable than those grown axenically. In each case, after approximately 18 generations of growth in axenic medium haploid amoebae predominated, while after 22 generations of growth on bacteria diploid amoebae still predominated over haploid amoebae.

In some cultures, in the process of haploidization, there was an unusual number of multinucleate amoebae. This must be taken into account when interpreting the data in Fig. 3, in which we totalled all the figures observed, whether they were in uninucleate, binucleate, or multinucleate amoebae. For example in DP27 in Expt I after nine generations of growth in axenic medium, 5I of 98 figures observed contained seven chromosomes. However, $75 \%$ of these haploids were in multinucleate amoebae while after 19 generations most of the haploid amoebae were uninucleate.

Figure 3 shows that aneuploid amoebae with all possible chromosome numbers between 7 and 14 were observed, and that aneuploids were most frequent during the period when the culture changes from being predominantly diploid to predominantly haploid. No class of aneuploids was found to be consistently enriched in cultures derived from diploid amoebae, and hence it is likely that the different classes of aneuploids occur at approximately the same frequency during haploidization of the cultures.

Analysis of spore size. Determination of the spore size from clones of amoebae isolated from the cultures at the same time that the amoebae were observed cytologically, verified the cytological observations with respect to haploid and diploid frequencies. In DP29, for example, after three generations in axenic medium all of 16 clones examined were diploid, but after ten generations, eight out of 20 clones examined were haploid. On the other hand, when DP26 was examined after seven generations in axenic medium, I 6 of 20 clones were haploid, one clone was diploid, and three clones had spores of varied sizes ranging from haploid to diploid. No attempt was made to measure spore size distribution so it is not known whether clones showing varied spore sizes were likely to be aneuploid on the basis of spore size (Sackin \& Ashworth, 1969). However, as yet no aneuploid clones have been observed when clones suspected of being aneuploid on the basis of spore size were examined cytologically; in all such cases the clones were shown to be haploid.

Analysis of plating efficiency and rates of growth. The plating efficiency and rates of growth of cells in the process of haploidization were examined to find out whether these characteristics are affected by the haploidization process. The plating efficiency (based on 200 to 400 clones analysed per strain) of DP26 through to DP29 varied between 27 and $36 \%$ after four generations of growth in axenic medium following transfer from a diploid clone grown on bacteria, and between $2 \mathrm{I}$ and $43 \%$ after seven generations in axenic medium. Strain X2, one of the parental strains, similarly transferred into axenic medium after growth on bacteria, was used as a control. Its plating efficiency was $71 \%$ after four generations and $96 \%$ after seven generations. By I 7 generations DP26 through to DP29 and X2 all showed high plating efficiency (between 50 and $100 \%$ ). The plating efficiency of the more stable bacterial diploid cultures was considerably higher than that observed for the axenic cultures. Hence plating efficiency was low during the period of haploidization.

The growth rate of the diploids in axenic medium after transfer from a diploid clone grown on bacteria was also slow compared with the haploid strain X2 also transferred from bacteria. After ten generations, however, the growth rate was similar. In bacterial cultures which were not haploid after 22 generations, the growth rate of diploid strains and strain $\mathrm{X} 2$ did not differ significantly for the 22 generations studied. Hence it seems that the growth rate of diploids is slower during the time of haploidization. 

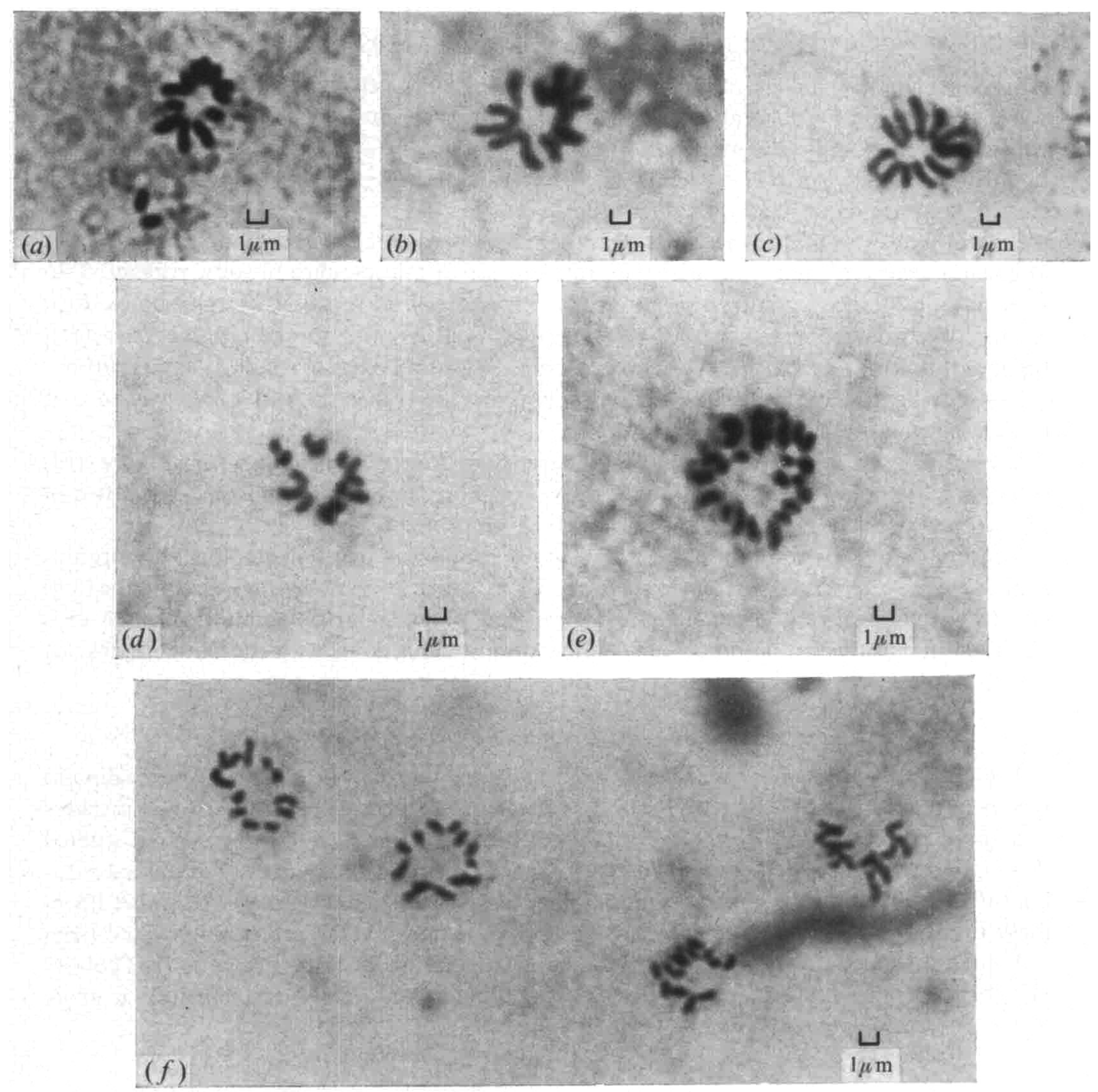

Fig. 4. Division figures present in cultures of diploid cells in the process of haploidization. (a) A cell containing 14 chromosomes in which two chromosomes have not been incorporated into the spindle. (b), (c) Cells illustrating somatic pairing, containing $(b) 12$ chromosomes and five discernible pairs and (c) I I chromosomes and four discernible pairs. (d) A cell with 15 chromosomes. $(e)$ A cell with at least 2I chromosomes. $(f)$ A tetranucleate cell; the two nuclei at either end contain I 2 chromosomes and the two central nuclei contain I3.

\section{Documentation of the presence of aneuploid cells in cultures of diploid cells}

In Fig. 2 division figures of all classes of aneuploid cells are shown. Figure $2(b)-(g)$ shows aneuploid cells with eight to thirteen chromosomes. Figure $2(b)$ is a tetranucleate cell: each nucleus has eight readily discernible chromosomes. In addition there is an isolated chromosome between the two nuclei on the right. 


\section{Cytological observations of aneuploid cells,}

Several phenomena were observed in cultures of diploid cells which may have a bearing on the mechanism for generation of haploid cells. Several mitotic figures were found which appeared to have stray chromosomes. Figure $4(a)$ shows one such mitotic figure, a cell with I 4 chromosomes, 12 of which are arranged in a ring and two of which are separated from the ring and are associated with each other.

Several figures were observed in which there appeared to be a non-random distribution of chromosomes in the metaphase plate reminiscent of mitotic chromosome pairing. Two such mitotic figures are shown. Figure $4(b)$ shows a cell with twelve chromosomes. Five groups of chromosome pairs may be present as well as two non-paired chromosomes. Figure $4(c)$ shows a cell with eleven chromosomes. Here four pairs of similarly sized chromosomes may be discerned. Because these observations are rather subjective, the existence of mitotic pairing must remain open to doubt.

Several mitotic figures were seen with more than fourteen chromosomes. Figure $4(d)$ shows a cell with 15 chromosomes while a cell with at least 21 chromosomes is shown in Fig. 4(e). Other cells contained 16,22 and 26 chromosomes.

Multinucleate cells were observed with figures containing different numbers of chromosomes, e.g. Fig. $4(f)$ shows a tetranucleate cell in which two of the figures contained I3, and two contained 12 chromosomes. However, the great majority of multinucleated cells observed in cultures in the process of haploidization had figures with the same number of chromosomes.

\section{Characterization of haploid clones derived from diploid strains}

The chromosome number was determined in clones that had been isolated from diploid strains and which had been characterized as haploid on the basis of chromosomal markers and spore size. Strain X2, a haploid strain derived from DP3 (Table I), was most studied (Fig. I) but ten other such clones isolated from four different diploids were examined cytologically. In all cases the chromosome number and size distribution were the same. From these results we conclude that resegregated haploid strains, which are easily isolated from a diploid strain, are normal haploid strains. The isolation of such strains is particularly valuable, since desirable characteristics of two parental strains can be combined in a single strain (for examples, see Williams et al. 1974).

\section{DISCUSSION}

This paper reports a reliable, rapid and simple fixing and staining procedure to facilitate the cytological analysis of Dictyostelium discoideum. We have been able to produce wellspread prophase chromosomes which should ultimately prove amenable to the complete resolution of the chromosomal complement, possible by using the procedures which have resulted in the identification of mammalian chromosomes (for review see Hsu, 1974). At this time we have distinguished two chromosomes on the basis of size. The largest chromosome, which has a light staining region, may be identical to the chromosome containing the nucleolar organizer described by Wilson (1953).

This paper is concerned with the parasexual cycle (Sinha \& Ashworth, 1969; Katz \& Sussman, 1972) and in particular with the questions of the stability of the diploid phase and the mechanism of haploidization. The results in this paper do not bear on the probability of a sexual cycle (macrocyst formation, see Clark, Francis \& Eisenberg, 1973; Erdos, 
Raper \& Vogen, 1973) since we have not attempted to study cytologically meiosis in macrocysts.

We have cytologically characterized clones of diploid cells and have analysed their chromosomal complement as a function of generations of growth. On the basis of the results obtained some comment about the stability of diploid strains seems necessary. There are contradictory statements concerning the stability of diploid strains in Dictyostelium discoideum. On the one hand diploid strains have been described as very 'unstable' (Sinha \& Ashworth, 1969), and on the other hand as 'stable' and 'metastable' (Sussman \& Sussman, 1963). In our examination of the literature we have compared the stability (incidence of haploids in the population) reported by each of the above authors and compared them with our results. The first diploids analysed for stability were by Sussman \& Sussman (1963). They reported that a diploid strain which they described as 'stable' could vary in its rate of haploidization in different experiments. In one culture of the 'stable' diploid strain RA, $80 \%$ haploidization occurred after 20 generations, while in another experiment with the same strain, the culture was less than $5 \%$ haploid after 20 generations. Sussman \& Sussman's (1963) 'metastable' strain I-2A had negligible numbers of haploids initially, and by 23 generations was 6 or $23 \%$ haploid (depending on the criterion used for ploidy). Sinha \& Ashworth (1969), on the other hand, found that after about 20 generations from a single clone (not 250 as reported in the paper) a diploid strain designated 'unstable' was predominantly haploid, containing at least $87 \%$ haploids (Ashworth, personal communication). When the known variation in the stability of a given strain is considered (Sussman \& Sussman, I963; Loomis, 1969) all of the strains mentioned above appear to have a similar stability. The stability of the diploids observed in this study falls within the same range as that reported above. It appears from our results that stability of diploids may vary under different culture conditions.

Thus we conclude that in general (there may be specific exceptions) the diploids of Dictyostelium discoideum, whether appearing spontaneously (for example strain RA, Sussman \& Sussman, 1963) or formed by using selective techniques (Loomis \& Ashworth, I968; Loomis, 1969; Sinha \& Ashworth, 1969; Katz \& Sussman, 1972; Williams et al. 1974), have similar stability which is variable because of the random nature of haploidization and possibly because of differences in conditions of growth. A similar stability is not entirely unexpected since all of the studies have been done on strains derived from a single basic isolate (strain NC-4). Significant haploidization usually (but not always) occurs in the first 20 generations after picking from a diploid clone. We intend to refer to such diploids as 'stable' since they can be maintained by clonal passage and are useful for genetic study. We have isolated 60 genetically different diploids of this type, and when allowances are made for experimental problems we have not found any strains which are unable to form 'stable' diploids. If there are 'unstable' diploids it is likely that they could not be isolated by the techniques currently employed, as these techniques require that a diploid has sufficient stability to generate a clone that is composed predominantly of diploid cells.

In the studies of parasexuality in Aspergillus nidulans by Kafer (I96I), non-disjunction was suggested as the initial step in the haploidization process, since aneuploids of the class $2 n+$ I were frequent. Haploidization takes place by a gradual loss of chromosomes until stable haploids are formed. Both $n+\mathrm{I}$ and $2 n+\mathrm{I}$ aneuploid strains of $\boldsymbol{A}$. nidulans disomic or trisomic for any one of the eight chromosomes have been isolated, although they are unstable and readily give rise to haploid and diploid sectors respectively (Kafer \& Upshall, 1973). Rare hyperhaploid strains $n+2, n+3$, and $n+4$ have been recovered, but these are even more unstable than disomics, while $n+5, n+6$ and $n+7$ hyperhaploids have never been 
recovered by plating, and Kafer (I96I) suggests that such aneuploids have not sufficient stability to be recovered on plating.

In comparison we have verified that aneuploid cells are intermediates in the haploidization of Dictyostelium discoideum (see Sinha \& Ashworth, 1969), and report that all classes of aneuploids exist. We conclude that at least the nuclei of aneuploids, and probably the cells themselves, can divide for a small number of generations, since in a number of cases multiple aneuploid mitoses were found in which the same number of chromosomes were present in each nucleus. We presume that these nuclei were derived from a single parent by division. We have seen metaphases supporting non-disjunction (Fig. $4 d$ ) and chromosome lag (Figs. $2 b, 4 a$ ), exclusion of chromosomes from the spindle, as possible mechanisms for the generation of aneuploids. It seems possible that in some cases more than one chromosome is lost at a division (Fig. $4 a$ ). In contrast to the situation in Aspergillus nidulans, we have not as yet isolated any aneuploid strains in $D$. discoideum; this aspect needs further investigation, particularly with further development of genetic techniques, since very slow growing clones (possibly aneuploid) have been observed in clonal plating of diploids in the process of haploidization. Aneuploids are clearly much less stable than diploids, since in some cases where aneuploids comprised in excess of $50 \%$ of the mitotic figures, on clonal isolation only haploid and diploid clones were observed.

Our findings are useful in understanding how changes in ploidy take place in Dictyostelium discoideum, and the results provide a cytological demonstration that the parasexual cycle can be used with confidence for the genetic analysis of this species.

The authors thank P. Newell and M. Bobrow for their interest in, and encouragement of our research. T. Brody is a postdoctoral fellow of The Helen Hay Whitney Foundation.

\section{REFERENCES}

Clark, M. A., Francis, D. \& Eisenberg, R. (1973). Mating types in cellular slime molds. Biochemical and Biophysical Research Communications 52, 672-678.

ERdos, G. W., RAPER, K. B. \& VoGen, L. K. (1973). Mating types and macrocyst formation in Dictyostelium discoideum. Proceedings of the National Academy of Sciences of the United States of America 7o, $1828-1830$.

Hamerton, J. L. (197I). Human Cytogenetics, vol. I. New York: Academic Press.

Hsu, T. C. (1974). Longitudinal differentiation of chromosomes. Annual Review of Genetics (in the Press).

KAFER, E. (I96I). The processes of spontaneous recombination in vegetative nuclei of Aspergillus nidulans. Genetics 46, $158 \mathrm{I}-\mathrm{I} 609$.

KafER, E. \& Upshall, A. (1973). The phenotypes of the eight disomics and trisomics of Aspergillus nidulans. Journal of Heredity 64, 35-38.

KATz, E. R. \& Sussman, M. (1972). Parasexual recombination in Dictyostelium discoideum: selection of stable diploid heterozygotes and stable haploid segregants. Proceedings of the National Academy of Sciences of the United States of America 69, 495-498.

LoomIs, W. F. (1969). Temperature-sensitive mutants of Dictyostelium discoideum. Journal of Bacteriology 99, 65-69.

Looms, W. F. (1971). Sensitivity of Dictyostelium discoideum to nucleic acid analogues. Experimental Cell Research 64, 484-486.

Loomis, W. F. \& Ashworth, J. M. (1968). Plaque-size mutants of the cellular slime mould Dictyostelium discoideum. Journal of General Microbiology 53, 18I-186.

Perkins, D. D. (1962). Preservation of Neurospora stock cultures with anhydrous silica gel. Canadian Journal of Microbiology 8, 591-594.

Ross, I. K. (1960). Studies on diploid strains of Dictyostelium discoideum. American Journal of Botany 47, 54-59. 
SACKIN, M. J. \& Ashworth, J. M. (I969). An analysis of the distribution of volumes amongst spores of the cellular slime mould Dictyostelium discoideum. Journal of General Microbiology 59, 275-284.

Sinha, U. \& AshwORTH, J. M. (1969). Evidence for the existence of elements of a parasexual cycle in the cellular slime mould Dictyostelium discoideum. Proceedings of the Royal Society B 173, 531-540.

Sussman, R. R. (196I). A method for staining the chromosomes of Dictyostelium discoideum myxamoebae in the vegetative stage. Experimental Cell Research 24, 154-155.

Sussman, R. R. \& Sussman, M. (1963). Ploidal inheritance in the slime mould Dictyostelium discoideum: haploidization and genetic segregation of diploid strains. Journal of General Microbiology 30, 349-355.

WATTS, D. J. \& AshworTH, J. M. (1970). Growth of myxamoebae of the cellular slime mould Dictyostelium discoideum in axenic culture. Biochemical Journal 119, 171-174.

Williams, K. L., Kessin, R. H. \& Newell, P. C. (1974). The genetics of growth in axenic medium of the cellular slime mould Dictyostelium discoideum. Nature, London 247, $142-143$.

Wilson, C. M. (1953). Cytological study of the life cycle of Dictyostelium. American Journal of Botany 40, 714-718. 